\title{
Effect of Magnetic Field on Mixed Convection Heat Transfer in a Lid-Driven Square Cavity
}

\author{
N. A. Bakar, ${ }^{1}$ A. Karimipour, ${ }^{2}$ and R. Roslan ${ }^{1}$ \\ ${ }^{1}$ Centre for Research in Computational Mathematics, Faculty of Science, Technology and Human Development, \\ Universiti Tun Hussein Onn Malaysia, 86400 Parit Raja, Batu Pahat, Johor, Malaysia \\ ${ }^{2}$ Department of Mechanical Engineering, Najafabad Branch, Islamic Azad University, Najafabad, Iran
}

Correspondence should be addressed to R. Roslan; rozaini@uthm.edu.my

Received 8 October 2015; Revised 1 February 2016; Accepted 14 February 2016

Academic Editor: Pedro Jorge Martins Coelho

Copyright (C) 2016 N. A. Bakar et al. This is an open access article distributed under the Creative Commons Attribution License, which permits unrestricted use, distribution, and reproduction in any medium, provided the original work is properly cited.

\begin{abstract}
The effect of magnetic field on fluid flow and heat transfer in two-dimensional square cavity is analyzed numerically. The vertical walls are insulated; the top wall is maintained at cold temperature, $T_{c}$ while the bottom wall is maintained at hot temperature, $T_{h}$ where $T_{h}>T_{c}$. The dimensionless governing equations are solved using finite volume method and SIMPLE algorithm. The streamlines and isotherm plots and the variation of Nusselt numbers on hot and cold walls are presented.
\end{abstract}

\section{Introduction}

Due to the effect on many engineering applications and nature phenomena, fundamental problem of combined free and forced convection has received considerable attention from researchers. Problem of heat transfer in low speed liddriven cavity is treated as a mixed convection problem. A forced convection conditions is created by the movement by one or two walls of the cavity while the temperature difference across the cavity caused a buoyancy driven flow. Hence, complicated heat and mass transfer flow occur inside the cavity. There have been many investigations in the past on mixed convection flow in lid-driven cavity. Many different configurations and combinations of thermal boundary conditions have been considered by various investigators. One of the earliest investigations on mixed convection in lid-driven cavity was conducted by [1]. Later, [2] studied numerically the effect of the Prandtl (Pr) number, on the laminar mixed convection heat transfer in a lid-driven cavity. The numerical simulations showed that, for higher values of Pr, the effect of thermal buoyancy force on the flow and heat transfer inside the cavity is more pronounced. Reference [3] investigated numerically mixed convection heat transfer in a driven cavity with a stable vertical temperature gradient. It was found that, for high values of the Richardson number, much of the fluid in both the middle and bottom portions of the cavity interior is stagnant. Also, results in [3] results showed that the flow features are similar to those of a conventional driven cavity of a nonstratified fluid for small values of the Richardson number. Meanwhile, [4] carried out experimental study on mixed convection heat transfer and fluid flow in a cavity where the upper lid was cooled and heated from bottom. Reference [5] examined numerically mixed convection flow in a lid-driven enclosure filled with a fluidsaturated porous medium and reported on the effects of the Darcy and Richardson numbers on the flow and heat transfer characteristics. Reference [5] observed that the Darcy number is most important parameter in convective flows. Moreover, the presence of internal heat generation provides an additional dynamic in overall convective flow system, which has significant influence on the features of isotherms and streamlines for small values of the Richardson number.

Subsequently, various investigations on mixed convection heat transfer in a lid-driven cavity are conducted. For instance, [6] investigated the mixed convection fluid flow and heat transfer in a square cavity with moving and differentially heated side walls. They found that the fluid flow and heat transfer in the cavity are affected by both, Richardson number 
and $\mathrm{Ri}$ and the direction of moving walls. Reference [7] investigated mixed convection fluid flow in a square cavity with linearly heated left wall, uniformly cooled bottom wall, insulated on the top while the right wall is either heated linearly or cooled uniformly. Reference [7] showed that by increase in Grashof number the strength of convection increased. Later, [8] investigated a uniformly heated bottom wall with linearly heated left side wall. Reference [9] analyzed four cases of mixed convection in a square cavity. For the first and second cases, the top and bottom walls are either heated or cooled while both side walls are insulated. In the third and fourth cases, the top and bottom walls are adiabatic while the side walls are either heated or cooled. Reference [9] reported that both $\mathrm{Ri}$ and direction of temperature gradient affect the flow pattern and heat transfer rate in the cavity. Mixed convection heat transfer and fluid flow in lid-driven cavities with different lengths of the heating portion and different location of it are studied by [10]. Reference [10] concluded that the heat transfer rate is enhanced on reducing the heating portion and when the portion is at the middle or top of the hot wall. Investigation of mixed convection fluid flow and heat transfer that combined linear and sinusoidal heating of different walls of a two-dimensional square cavity with a moving lid had been done by [11]. The effects of inclination angle, $\mathrm{Ri}$, and various aspects ratios on the flow and heat transfer in an air-filled cavity had been discussed by [12]. Reference [12] reported that, among the three aspects ratio investigated, the increase of inclination angle does not affect the flow structures and heat transfer when the flow is in a forced convection dominated regime.

Investigations on the significance of magnetic field on the fluid flow and performance of different systems have been growing through these past decades. Reference [13] studied the effects of magnetic field on free convection in a rectangular cavity. Then, [14] investigated the significance of transverse magnetic field in tilted enclosure numerically. Different thermal boundary conditions with the existence of magnetic field on natural convection are discussed by $[15,16]$. Recently, [17-19] investigated natural convection heat transfer and fluid flow in a cavity filled with nanofluid using the Lattice Boltzmann method. Reference [17] studied the effects of magnetic field to the natural convection flow in a rectangular cavity. Reference [18] investigated the MHD natural convection with sinusoidal temperature distribution on the right wall. Later, [19] extended the work by [18] when sinusoidal temperature distribution is applied on both side walls. Reference [20] examined the natural convection in a square enclosure that is filled with nanofluid and influenced by a magnetic field. Reference [20] reported that the values of Hartmann number and Rayleigh number influence the heat transfer performance for the increment of solid volume fraction. Very recently, [21] investigated the effects of magnetic field on natural convection of nanofluid in a threedimensional cubic cavity that is heated from below. It is reported that the applied magnetic field results in opposite force to the flow direction which leads to dragging the flow and then reduces the convection by reducing the velocities.

Reference [22] investigated combined convection flow in a two-dimensional square cavity filled with an electrically conducting fluid. Reference [22] also studied the effects of magnetic field and internal heat generation or absorption to the opposing and aiding flow. It was found that the presence of magnetic field strongly affected the heat transfer and flow behaviors. Very recently, the significance of both internal heating or absorption and magnetic field to the fluid flow and heat transfer rate in a lid-driven cavity is studied by [23]. Reference [23] showed that, by increasing the strength of magnetic field, the average Nusselt number dropped significantly. In addition, the effect of heat generation or absorption became insignificant for forced convection region. Then, [24] reported on the conjugate effect of Joule heating and magnetic field in an obstructed lid-driven cavity to the flow and heat transfer characteristics. It is found that the obstacle strongly affected the thermal field at forced convection regime and the flow field at mixed convection regime.

References [25, 26] studied the effects of different heating locations with the presence of magnetic field in a lid-driven cavity to the flow and heat transfer rate. They reported that heating location and existence of magnetic field have significant role in the heat transfer rate and fluid flow behavior. Hydromagnetic effects on mixed convection in a lid-driven cavity are studied by [27, 28]. Reference [27] investigated the effects with sinusoidal temperature on both side walls while [28] investigated them with sinusoidal corrugated bottom surface cavity. Both studies reported that magnetic field plays important role in the convection heat transfer rate and fluid flow behaviors. Later, [29] extended the work of [27] and studied the effects of both magnetic field and inclination angle on the mixed convection heat transfer and flow behaviors. The results showed that heat transfer rate is enhanced by increasing either the Hartmann number or inclination angle. References $[30,31]$ studied magnetohydrodynamic mixed convection in a lid-driven cavity with linearly heated wall. Very recently, [32] investigated the effects of magnetohydrodynamic mixed convection over an isothermal circular cylinder in the presence of an aligned magnetic field. The problems of mixed convection of a nanofluid in a differentially heated lid-driven cavity with the presence of magnetic field is investigated by $[33,34]$. Using different type of nanofluid, both researches reported that the heat transfer rate increased with an increase of the Reynolds number but it decreased with an increase of the Hartmann number. Then, [35] executed a numerical investigation of mixed convection in a nanofluid-filled cavity under the influence of magnetic field. Reference [35] compared the results obtained from the Maxwell method and modified Maxwell method. The results showed that heat transfer is higher based on the modified Maxwell method and the heat transfer mechanism is strongly dependent on the strength of magnetic field. Recently, [36] reported on mixed convection heat transfer of nanofluid flow in a vertical channel with sinusoidal walls under magnetic field effect. It is found that the average Nusselt number and Poiseuille number increased with an increase of the Hartman number. Numerical investigation of magnetic field on mixed convection and entropy generation of nanofluid in a trapezoidal enclosure was done by [37]. The results showed that, with imposing the magnetic field and enhancing it, the nanofluid convection and the strength of flow decrease and 


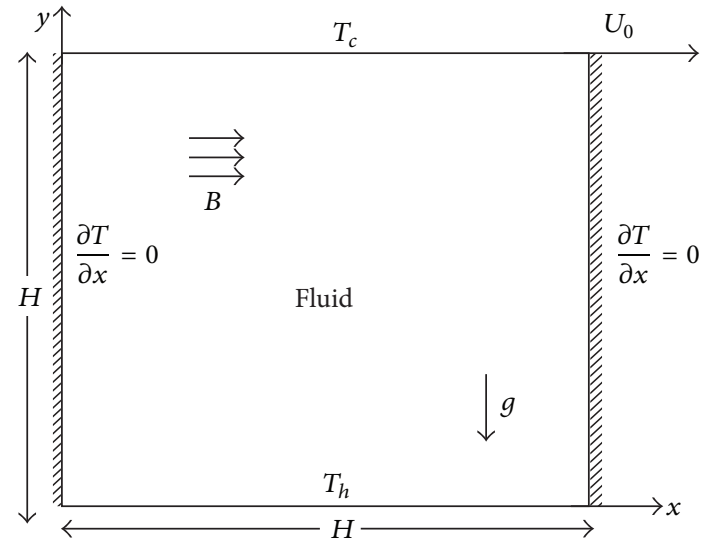

FIGURE 1: Geometrical configuration.

the flow tend toward natural convection and finally toward pure conduction. Thus, for all of the considered Reynolds numbers and volume fractions, the average Nusselt number decreased with an increase of the Hartmann number.

Based on the literature review, no work has been dealt on mixed convection flow and heat transfer in a lid-driven square cavity with the presence of magnetic field. Thus, the main objective of present work is to examine the influence of Hartmann number and the Richardson number on the characteristics of mixed convection flow inside a square cavity having the top lid moving with uniform speed.

\section{Mathematical Modelling}

Two-dimensional square cavity is illustrated in Figure 1. The width and height of the cavity are denoted as $H$. Both of the vertical walls are insulated while the bottom and top walls are maintained at constant and different temperature, $T_{h}$ and $T_{c}$, respectively, such that $T_{h}>T_{c}$. Furthermore, the top lid is assumed to have a constant speed $U_{0}$ that slides in the positive direction of $x$-axis. A uniform magnetic field of strength $B_{0}$ is applied in the horizontal direction normal to the adiabatic wall. The induced magnetic field due to the motion of the electrically conducting fluid is neglected in comparison to the external magnetic field. Moreover, the imposed and induced electrical fields are assumed to be negligible. The Joule heating of the fluid and the effect of viscous dissipation are also negligible. The working fluid is assumed to be Newtonian, incompressible, unsteady, and laminar flow. The gravitational force acts vertically downward. It is assumed that the thermophysical properties of the fluid are constant except the density in the body force term of the momentum equations which varies according to the Boussinesq approximation. Hence, based on the aforementioned assumptions, the dimensional forms of the continuity, momentum, and energy equations are as follows [25]:

$$
\begin{gathered}
\frac{\partial u}{\partial x}+\frac{\partial v}{\partial y}=0 \\
\frac{\partial u}{\partial t}+u \frac{\partial u}{\partial x}+v \frac{\partial u}{\partial y}=-\frac{1}{\rho} \frac{\partial p}{\partial x}+v\left(\frac{\partial^{2} u}{\partial x^{2}}+\frac{\partial^{2} u}{\partial y^{2}}\right),
\end{gathered}
$$

$$
\begin{aligned}
\frac{\partial v}{\partial t}+u \frac{\partial v}{\partial x}+v \frac{\partial v}{\partial y}= & -\frac{1}{\rho} \frac{\partial p}{\partial y}+v\left(\frac{\partial^{2} v}{\partial x^{2}}+\frac{\partial^{2} v}{\partial y^{2}}\right) \\
& +g \beta\left(T-T_{c}\right)-\frac{\sigma B_{0}^{2}}{\rho} v \\
\frac{\partial T}{\partial t}+u \frac{\partial T}{\partial x}+v \frac{\partial T}{\partial y}= & \alpha\left(\frac{\partial^{2} T}{\partial x^{2}}+\frac{\partial^{2} T}{\partial y^{2}}\right),
\end{aligned}
$$

where $x$ and $y$ are the coordinate directions and $t$ is the time. The variables $u, v, P$, and $T$ are the velocity components of the fluid in the $x$ - and $y$-directions, the pressure, and the temperature, respectively. The parameters $\beta, g, \sigma, \nu$, and $\rho$ are the fluid thermal expansion coefficient, the gravity, the electrical conductivity, the kinematic viscosity, and the density, respectively. Parameter $\alpha=k / \rho c$ is the thermal diffusivity, $k$ is the thermal conductivity, $c$ is the specific heat capacity, and $B_{0}$ is the magnetic induction coefficient. The initial and boundary conditions of the problem are as follows:

$$
\text { Top wall: } \begin{aligned}
u & =U_{0}, \\
v & =0, \\
T & =T_{c},
\end{aligned}
$$

Bottom wall: $u=v=0$,

$$
T=T_{h},
$$

Left and right walls: $u=v=0$,

$$
\frac{\partial T}{\partial x}=0 .
$$

Then, (1) are transformed to dimensionless equations using the following variables:

$$
\begin{aligned}
X & =\frac{x}{H}, \\
Y & =\frac{y}{H}, \\
U & =\frac{u}{U_{0}}, \\
V & =\frac{v}{U_{0}}, \\
\theta & =\frac{T-T_{c}}{T_{h}-T_{c}}, \\
\tau & =\frac{t U_{0}}{H}, \\
\operatorname{Gr} & =\frac{g \beta\left(T_{h}-T_{c}\right) H^{3}}{\nu^{2}}, \\
\operatorname{Pr} & =\frac{\nu}{\alpha}, \\
P & =\frac{p}{\rho U_{0}^{2}},
\end{aligned}
$$



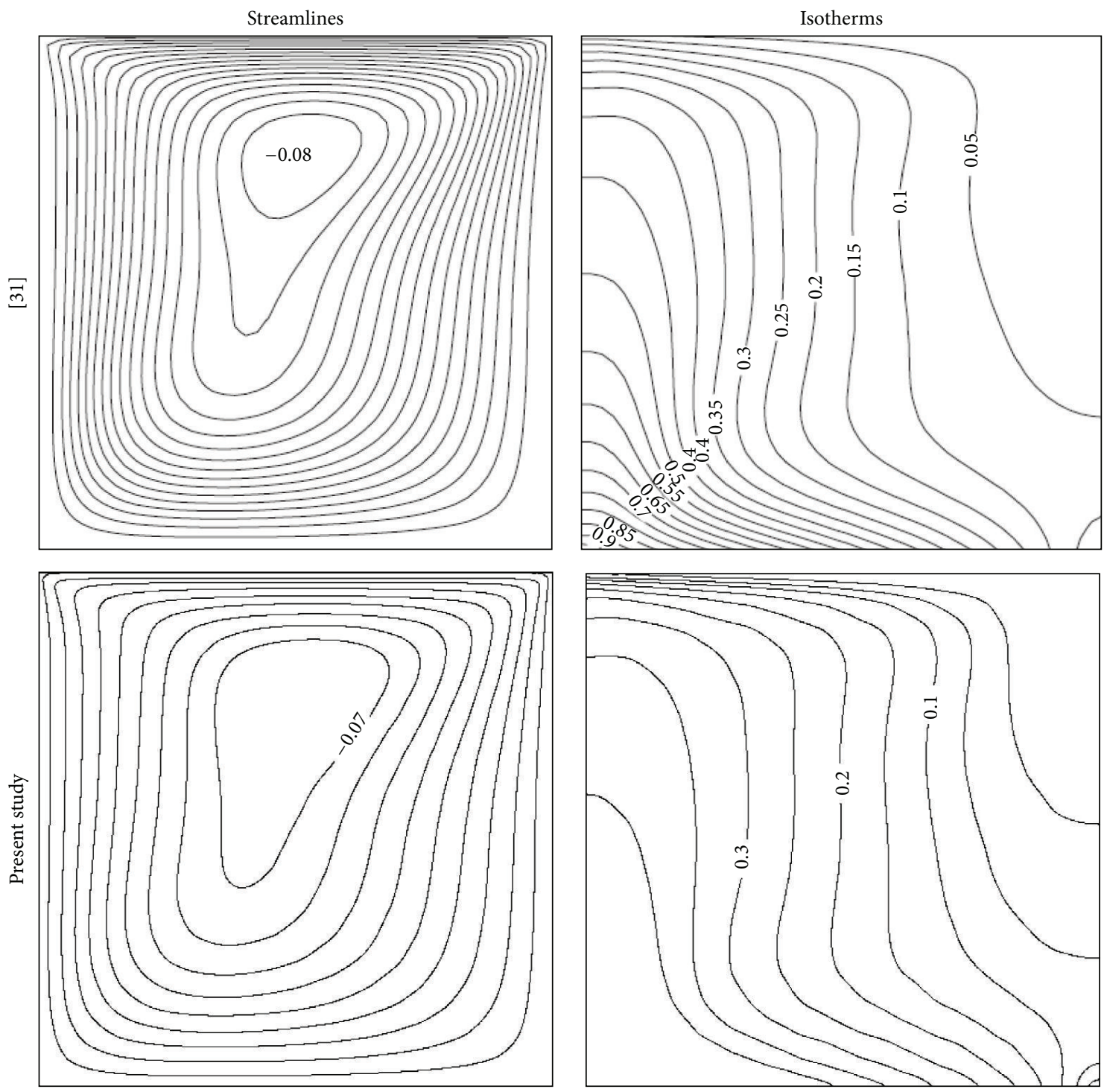

FIgURE 2: Comparison of present results with [31].

$$
\begin{gathered}
\mathrm{Re}=\frac{U_{0} H}{v}, \\
\mathrm{Ha}^{2}=\frac{B_{0}^{2} H^{2} \sigma}{\rho v},
\end{gathered}
$$

$$
\begin{aligned}
& \frac{\partial U}{\partial \tau}+U \frac{\partial U}{\partial X}+V \frac{\partial U}{\partial Y}=-\frac{\partial P}{\partial X}+\frac{1}{\operatorname{Re}}\left(\frac{\partial^{2} U}{\partial X^{2}}+\frac{\partial^{2} U}{\partial Y^{2}}\right) \\
& \frac{\partial V}{\partial \tau}+U \frac{\partial V}{\partial X}+V \frac{\partial V}{\partial Y}=-\frac{\partial P}{\partial Y}+\frac{1}{\operatorname{Re}}\left(\frac{\partial^{2} V}{\partial X^{2}}+\frac{\partial^{2} V}{\partial Y^{2}}\right)
\end{aligned}
$$

where $\theta, \tau$, and $P$ are the nondimensional temperature, the time, and the pressure, respectively. Parameters Gr, Re, Pr, and $\mathrm{Ha}^{2}$ are the Grashof number, the Reynolds number, the Prandtl number, and the Hartmann number, respectively. Substituting the variables into the governing equations, we obtain the following dimensionless equations:

$$
\frac{\partial U}{\partial X}+\frac{\partial V}{\partial Y}=0
$$$$
+\frac{\mathrm{Gr}}{\mathrm{Re}^{2}} \theta-\frac{\mathrm{Ha}^{2}}{\mathrm{Re}} V,
$$$$
\frac{\partial \theta}{\partial \tau}+U \frac{\partial \theta}{\partial X}+V \frac{\partial \theta}{\partial Y}=\frac{1}{\operatorname{Re} \operatorname{Pr}}\left(\frac{\partial^{2} \theta}{\partial X^{2}}+\frac{\partial^{2} \theta}{\partial Y^{2}}\right) .
$$ 

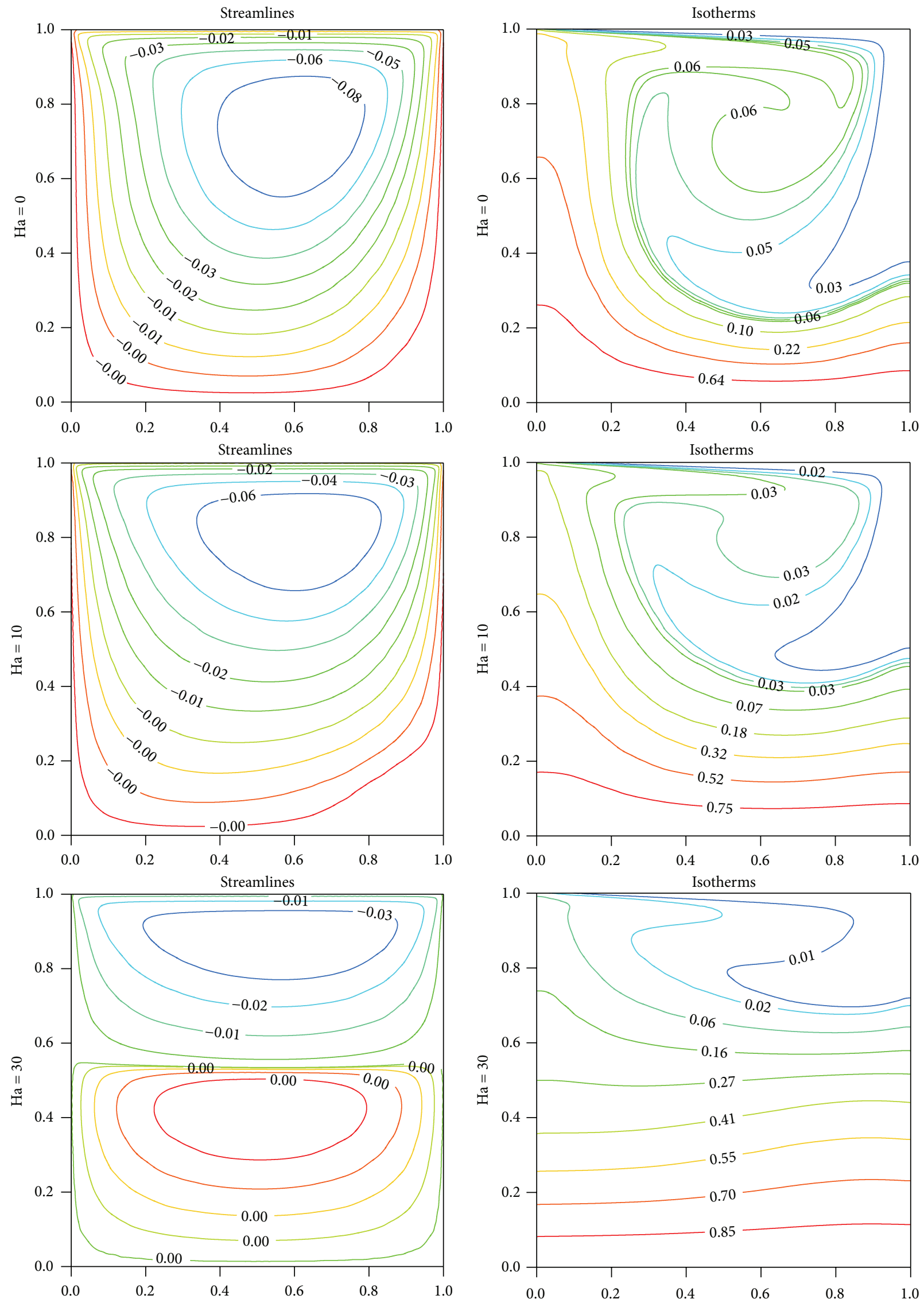

Figure 3: Continued. 

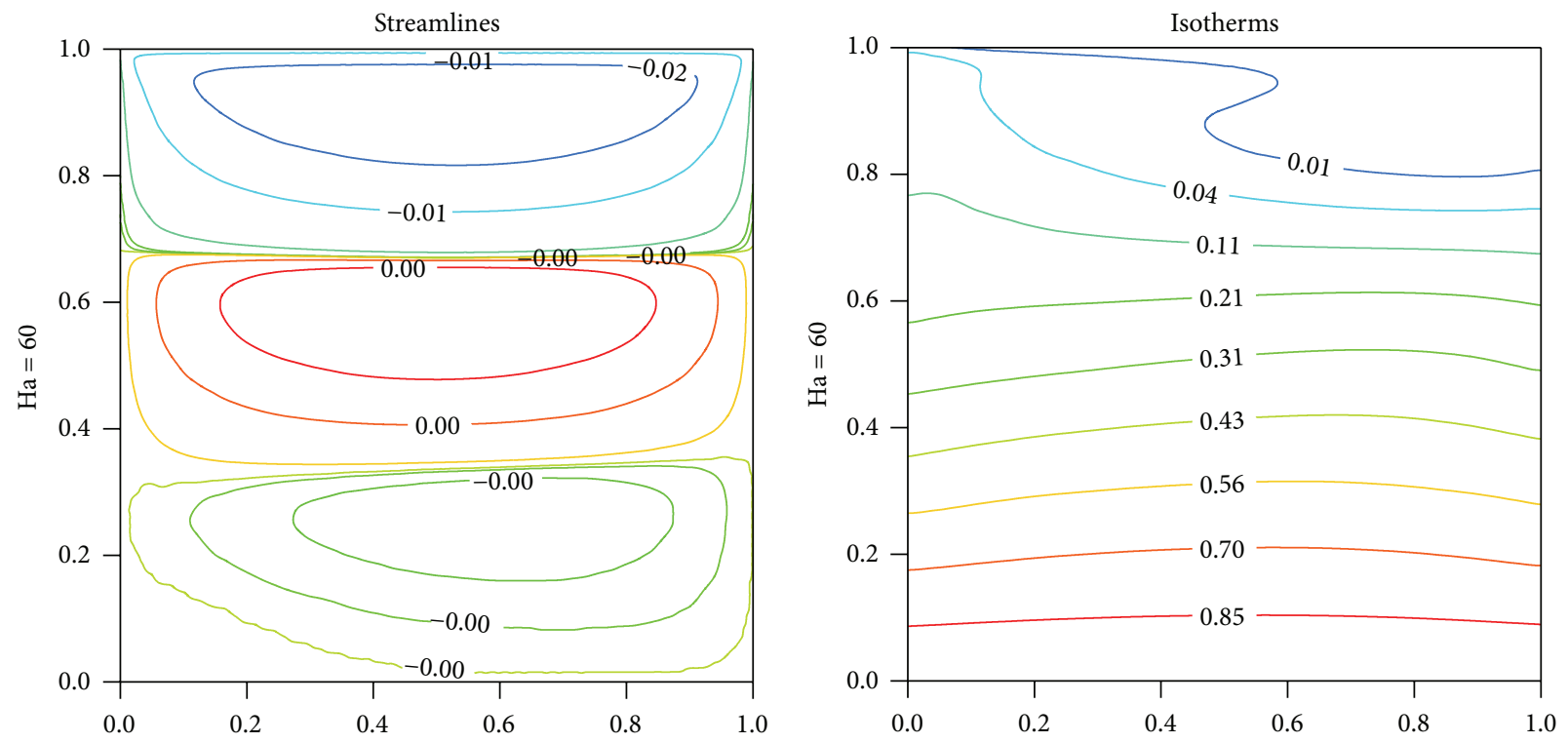

FIgURE 3: Streamlines and isotherms with $\mathrm{Ri}=0.1, \mathrm{Gr}=10^{3}, \mathrm{Re}=100.0$, and different values of Hartmann number.

The dimensionless boundary conditions can be written as

$$
\text { Top wall: } \begin{array}{r}
U=1, \\
V=0, \\
\theta=0,
\end{array}
$$

Bottom wall: $U=V=0$,

$$
\theta=1 \text {, }
$$

Left and right wall: $U=V=0$,

$$
\frac{\partial \theta}{\partial X}=0
$$

\section{Numerical Method}

The finite volume method [38] is used to solve the governing equations (4) subject to the boundary conditions (5). The convection and diffusion terms are discretized by the power law. The resulting set of algebraic equations are solved iteratively using tridiagonal matrix algorithm (TDMA). In order to couple the pressure and velocity field in the momentum equations, the SIMPLE algorithm is adopted. The numerical solutions are obtained by choosing a staggered grid system, such that the velocity components are stored halfway between the scalar storage locations. Then, the unknown $u, v$, and $\theta$ are calculated iteratively until the following convergence criterion is fulfilled:

$$
\varepsilon=\frac{\sum_{j=1}^{m} \sum_{i=1}^{n}\left|\zeta_{i, j}^{k+1}-\zeta_{i, j}^{k}\right|}{\sum_{j=1}^{m} \sum_{i=1}^{n}\left|\zeta_{i, j}^{k+1}\right|} \leq 10^{-7}
$$

TABLE 1: The average Nusselt number and the number of grids for $\mathrm{Ri}=1.0$ and $\mathrm{Ha}=60$.

\begin{tabular}{lc}
\hline Grid & Average Nusselt number, $\overline{\mathrm{Nu}}$ \\
\hline $61 \times 61$ & 1.26228 \\
$81 \times 81$ & 1.27974 \\
$101 \times 101$ & 1.28140 \\
$121 \times 121$ & 1.29458 \\
$141 \times 141$ & 1.31062 \\
\hline
\end{tabular}

where $\varepsilon$ is the tolerance; $m$ and $n$ are the number grid points in $x$ - and $y$-directions, respectively; $\zeta$ is any of the computed field variables; and $k$ is the iteration number. The heat transfer rate is obtained using the Nusselt number. The local Nusselt number along the hot wall is defined as $\mathrm{Nu}_{\text {local }}=$ $-(\partial \theta / \partial Y)_{Y=0}$ and the average Nusselt number is expressed as follows:

$$
\overline{\mathrm{Nu}}=\int_{0}^{1} \mathrm{Nu}_{\mathrm{local}} d X .
$$

To find the field variables grid-independency solutions, the grid sensitivity tests are conducted. The test is performed for $\mathrm{Ri}=1.0, \mathrm{Pr}=6.2$, and $\mathrm{Ha}=60$ in a top cooled moving lid, bottom heated, and vertically insulated cavity. The Nusselt numbers along the hot bottom wall are compared for the grid sizes from $61 \times 61$ to $141 \times 141$. Based on the results presented in Table 1 , a grid size $81 \times 81$ is enough to solve the system of equations.

The present code using the Fortan 90 programming language is validated against published work of $[3,5,31]$. Streamlines and isotherms are plotted in Figure 2 for $\mathrm{Re}=$ $100, \mathrm{Gr}=10^{5}, \mathrm{Ha}=30.0$, and the lid moves from left to 

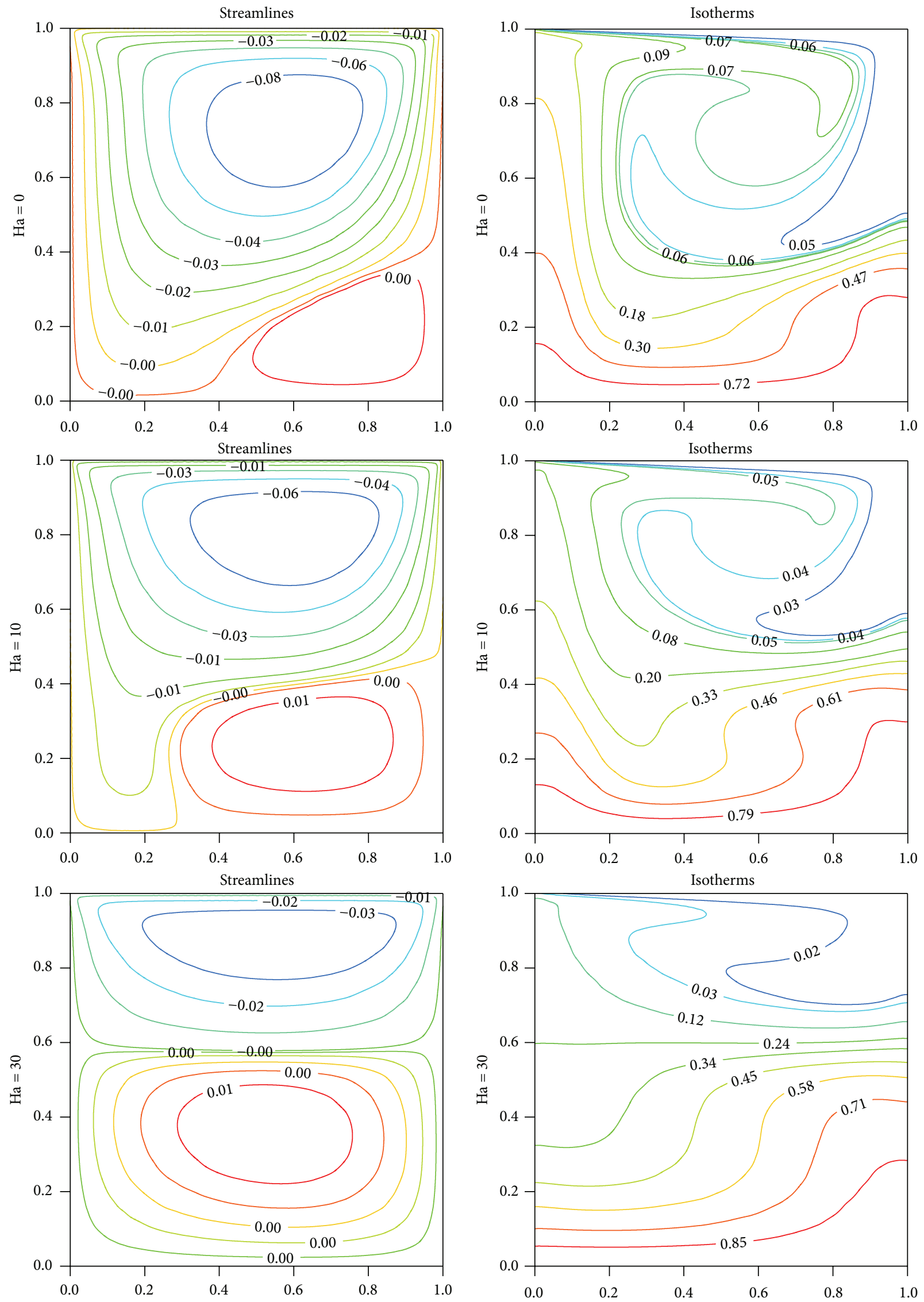

FIgURE 4: Continued. 

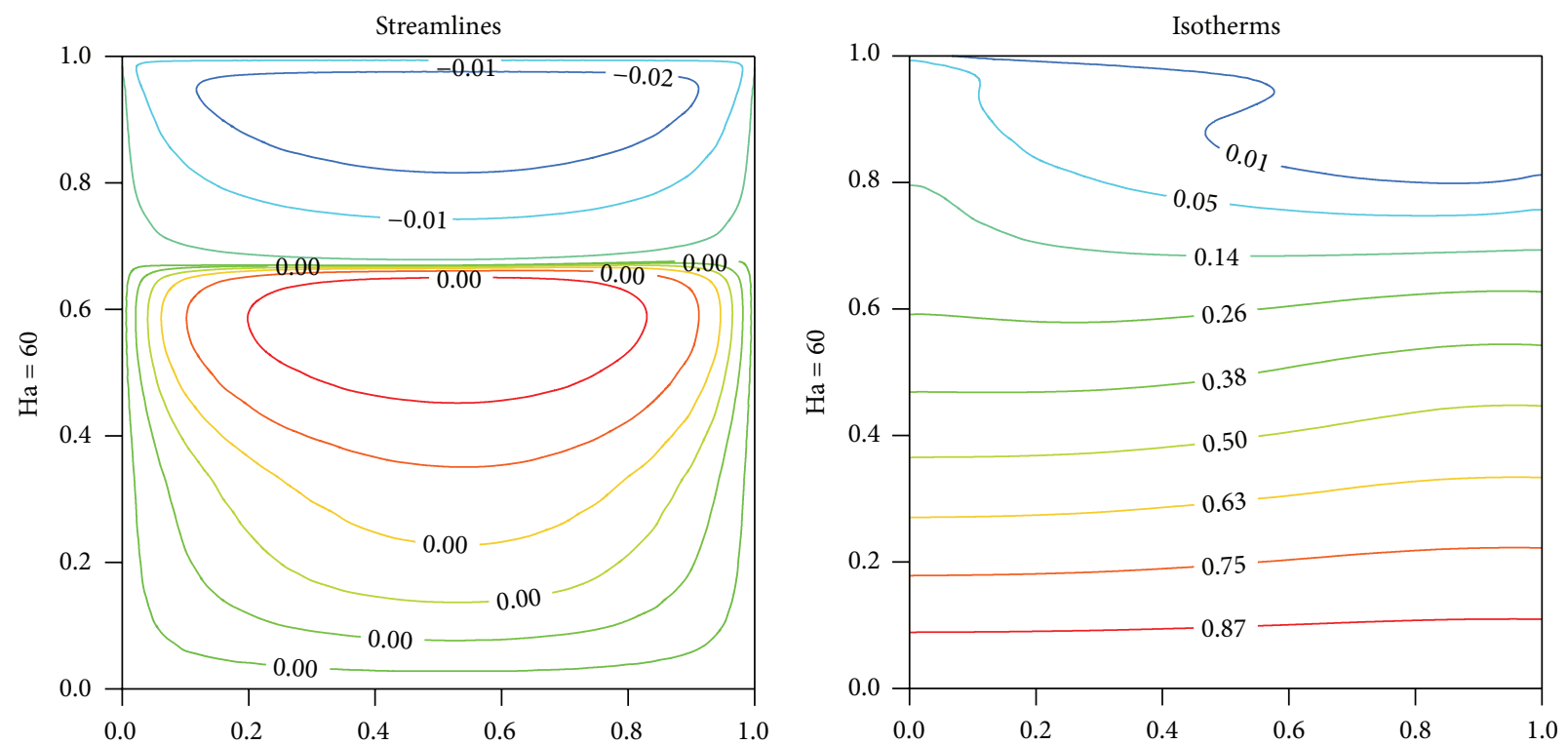

FIGURE 4: Streamlines and isotherms with $\mathrm{Ri}=1.0, \mathrm{Gr}=10^{4}, \mathrm{Re}=100.0$, and different values of Hartmann number.

TABle 2: Comparisons of the maximum and minimum values of the horizontal and vertical velocities at the midsection of the cavity between present solution and the works by $[3,5]$.

\begin{tabular}{lccc}
\hline & {$[3]$} & {$[5]$} & Present \\
\hline$U_{\min }$ & -0.3197 & -0.3099 & -0.3023 \\
$U_{\max }$ & 1.000 & 1.0000 & 1.0000 \\
$V_{\min }$ & -0.4459 & -0.4363 & -0.4219 \\
$V_{\max }$ & 0.2955 & 0.2866 & 0.2802 \\
\hline & & $\mathrm{Re}=100$ & \\
$U_{\min }$ & -0.2122 & -0.2037 & -0.2049 \\
$U_{\max }$ & 1.0000 & 1.0000 & 1.0000 \\
$V_{\min }$ & -0.2506 & -0.2448 & -0.2328 \\
$V_{\max }$ & 0.1765 & 0.1699 & 0.1673 \\
\hline
\end{tabular}

right. From Figure 2, it is clear that the plots between the two numerical results are in good agreement. Furthermore, the present code is validated against published works in the literature on convective flow in lid-driven cavities $[3,5]$. The results are compared in Table 2 and show a good agreement.

\section{Results and Discussion}

The mixed convection heat transfer and fluid flow for a liddriven square cavity with the presence of magnetic field are numerically investigated. Both vertical walls are perfectly insulated while the bottom and top walls are maintained at different temperature. In the present analysis, water is chosen as the working fluid with $\operatorname{Pr}=6.2$. The controlling parameters are Richardson number, $\mathrm{Ri}=\mathrm{Gr} / \mathrm{Re}^{2}$, and Hartmann number (Ha). Reynolds number is kept fixed at $\mathrm{Re}=100$ and
Grashof number is varied in the range from $10^{3}$ to $10^{5}$. The values of magnetic field parameter analyzed are $\mathrm{Ha}=0$, 10,30 , and 60 . The results are presented in streamlines and isotherms plot at three different regimes that are forced convection $(\mathrm{Ri}=0.1)$, mixed convection $(\mathrm{Ri}=1.0)$, and natural convection $(\mathrm{Ri}=10.0)$.

The streamlines and isotherms for different values of $\mathrm{Ha}$ for $\mathrm{Ri}=0.1$ are presented in Figure 3. In the absence of magnetic field, streamline plot indicated that the shear effect is dominant. This is due to the movement of the top lid. A primary clockwise recirculating eddy characterized the fluid flow with $\mathrm{Ha}=0$ such that the flow is driven by the movement of top lid and is forced to move downward. The primary recirculating eddy then tends to move closer to the moving wall by adding the strength of magnetic field such that $\mathrm{Ha}=$ 10. As $\mathrm{Ha}$ increases to 30 , a counter-clockwise recirculating eddy is developed along the bottom wall. Another clockwise recirculating eddy developed by increasing $\mathrm{Ha}$ to 60 . The temperature contours illustrated a steep temperature gradient along the center of top wall and right wall in the absence of magnetic field. This is due to domination of force convection and shear force of the top lid. As magnetic field strength increases, the temperature gradient mentioned earlier is diminished. The isotherms show that the fluid is horizontally stratified at the bottom wall at $\mathrm{Ha}=30$. The temperature gradient is parallel to the horizontal wall at $\mathrm{Ha}=60$. This implies that the heat is transferred by conduction.

For the mixed convection regime with $\mathrm{Ri}=1.0$, the streamlines and isotherms plots are presented in Figure 4. The streamlines show that a primary clockwise recirculating eddy occupied most of the cavity while a secondary counterclockwise eddy exists at the right bottom corner. By increasing the magnetic field strength, the secondary eddy gradually became bigger and the primary eddy became smaller. The 

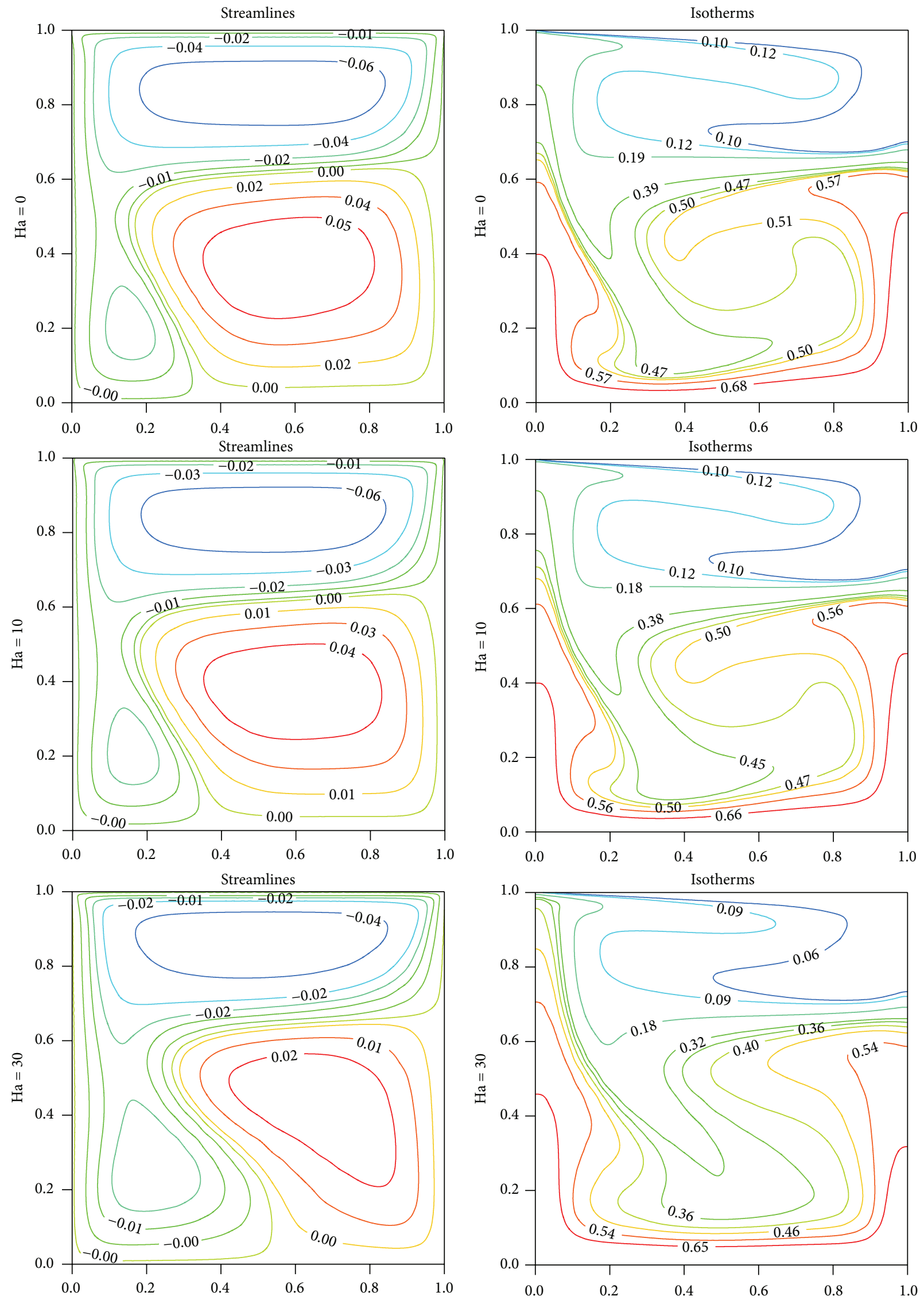

Figure 5: Continued. 

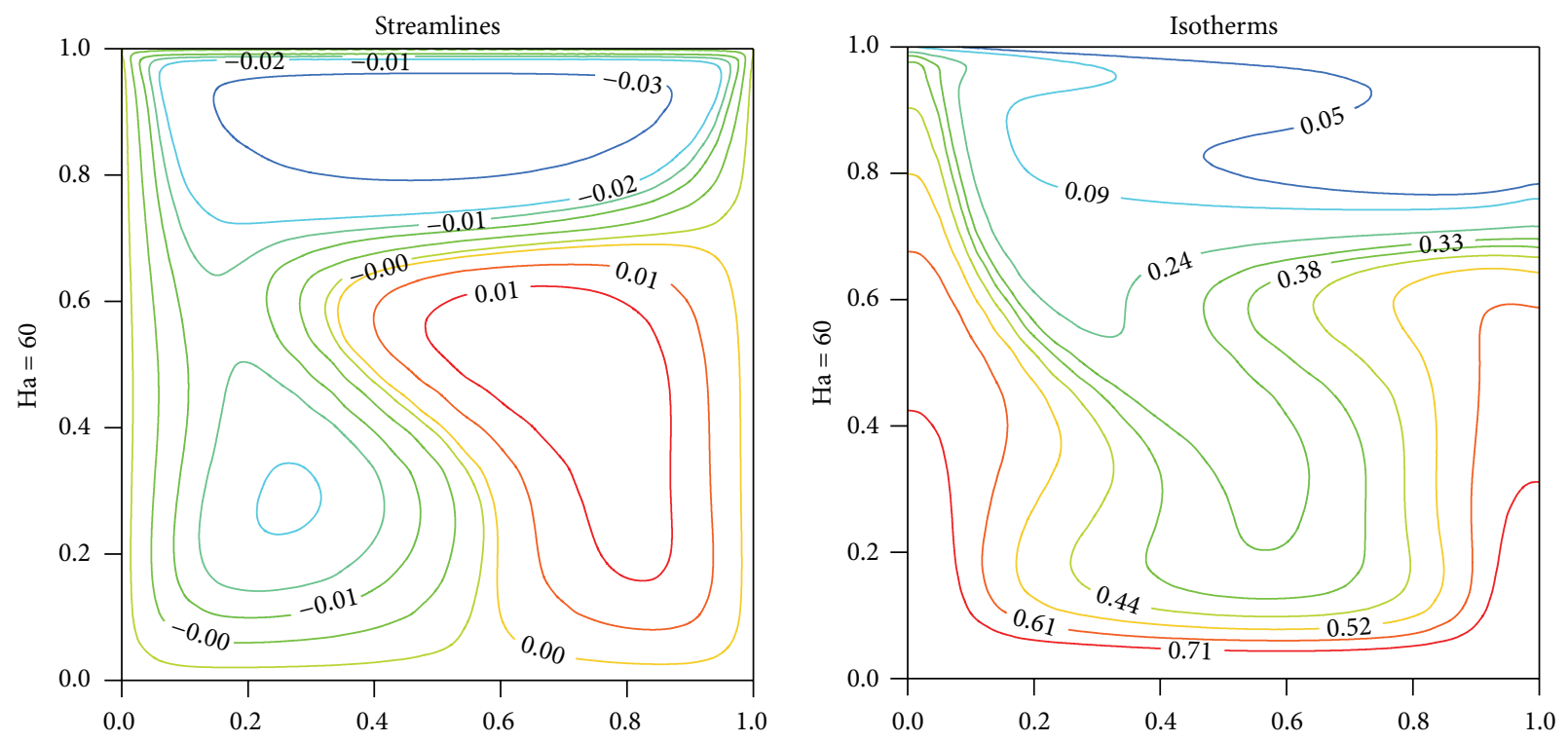

FIGURE 5: Streamlines and isotherms with $\mathrm{Ri}=10.0, \mathrm{Gr}=10^{5}, \mathrm{Re}=100.0$, and different values of Hartmann number.

isotherms show that the effect of magnetic field is suppressing the convective heat transfer mechanism. This is due to uniformly distributed isotherms in the cavity especially at the bottom.

Figure 5 shows the streamlines and isotherms for natural convection regime. There are three recirculating eddies which appear when there is no magnetic field presence for $\mathrm{Ri}=10.0$. However, only two major recirculating eddies occupied the cavity. The minor eddy located at the left bottom corner is attached to the major clockwise recirculating eddy at top while the eddy at the right bottom wall is recirculating in counter-clockwise direction. Increasing the Hartmann number causes the major recirculating eddy at the top wall gradually to become larger and occupy the cavity. The flow strength is weakening as the magnetic field is strengthening. From Figure 5, the variations of Hartmann numbers made big impact on the isotherms. At higher Hartmann number, the isotherms are almost vertical to the adiabatic walls and stratified at the bottom of the cavity. This indicates that convective flow is weaker and less heat transfer occurs.

Figure 6 shows the velocity profile along the vertical direction at the midaxis in $x$-direction at different Hartmann numbers. Figure 6(a) presents that the velocity magnitude decreases with an increase of Hartmann number for $\mathrm{Ri}=$ 0.1 . In similar manner, Figure $6(\mathrm{~b})$ shows that the velocity profile decreases with an increase of Hartmann number, with $\mathrm{Ri}=1.0$. Minimum and maximum points are observed due to clockwise and counter-clockwise flows. Again, a decreasing effect of velocity magnitude is observed in Figure 6(c) for Ri = 10.0. It is clearly seen that magnetic field retards the velocity and the trend of velocity changes with Hartmann number and Richardson number. The effects of Hartmann number on local Nusselt number at the bottom wall have been studied as seen in Figure 7. The results are showing that the maximum local Nusselt number increases as the Richardson number increases. It decreases as the Hartmann number increases due to the suppression of the convective flow.

The effects of Richardson number and the Hartmann number on the average Nusselt number are presented in Figure 8. Overall, the average Nusselt number decreases with the increase of the Hartmann number. The main characteristics of flow and temperature in the cavity for $\mathrm{Ri}=0.1$, 1.0, or 10.0 are compared to Figures $3-5$ to elucidate the cause of heat transfer drop. A main clockwise recirculating vortex occupies the cavity and a minor counter-clockwise recirculating vortex appears at the bottom corner and at right bottom corner of the cavity for $\mathrm{Ri}=0.1, \mathrm{Ri}=1.0$, and $\mathrm{Ri}=$ 10.0, respectively. As the magnetic field strength increases, the minor vortex starts to grow bigger and the primary recirculating vortex tends to move closer to the moving wall. In addition, the effect of magnetic field is suppressing the convective heat transfer mechanism that the isotherms become more parallel to the moving wall indicating a quasiconduction regime. This illustrates the average heat transfer decreases as the magnetic field increases as shown in Figure 8. This is due to the negative effect of magnetic field on the buoyancy force. By increasing the magnetic field strength, the effect of convection is decreased. Hence, the flow motion reduces resulting conduction as the dominant heat transfer mechanism.

\section{Conclusions}

In the present study, mixed convection in a lid-driven cavity filled with water in the presence of magnetic field is studied numerically by employing the finite volume method. The left and right walls are perfectly insulated. The top lid is moving uniformly from left to right while the bottom wall is heated with constant temperature. The effects of $\mathrm{Ri}$ and $\mathrm{Ha}$ on the fluid flow and heat transfer behavior are 


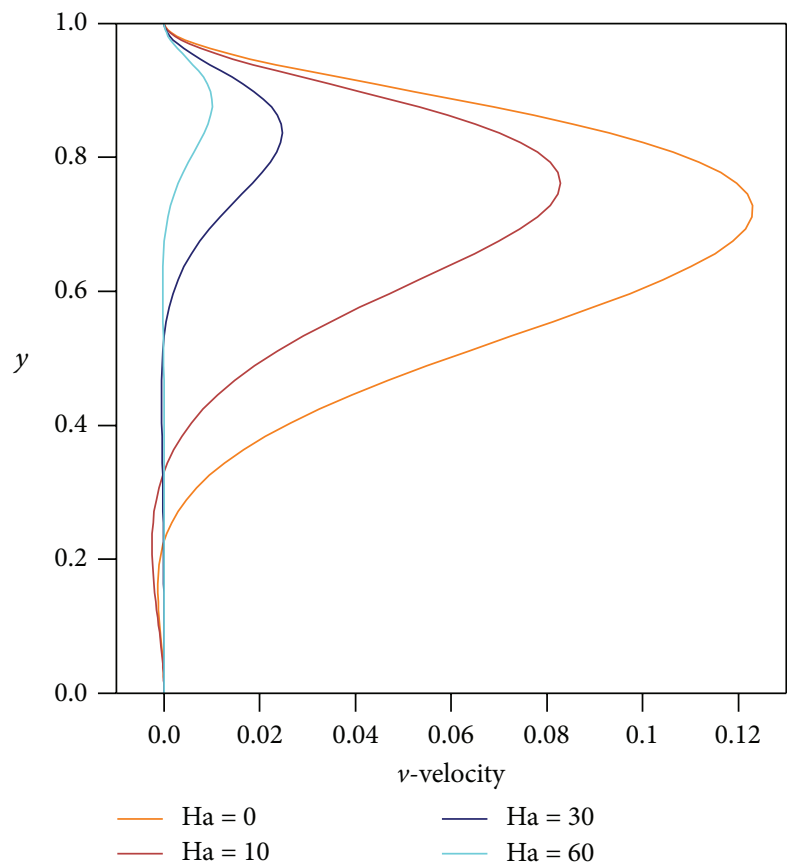

(a) $\mathrm{Ri}=0.1$

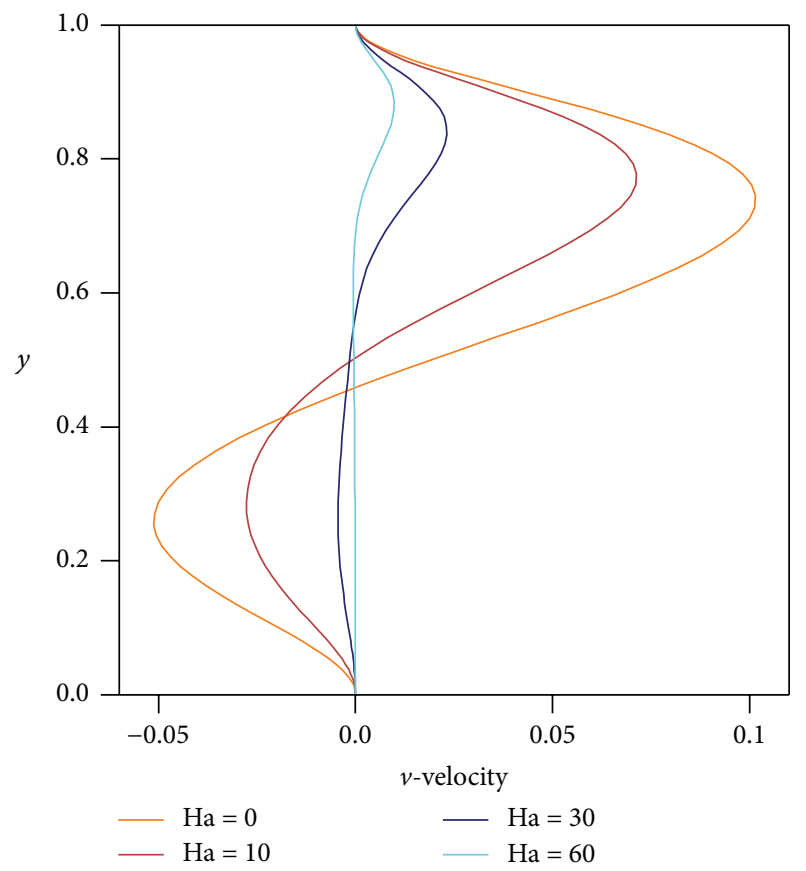

(b) $\mathrm{Ri}=1.0$

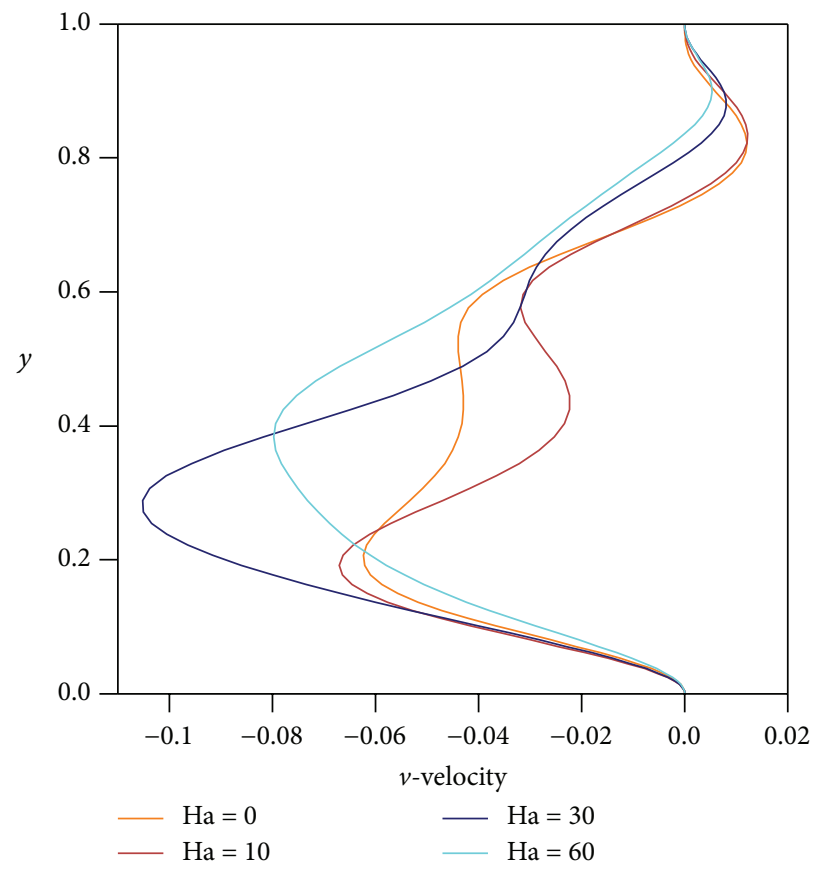

(c) $\mathrm{Ri}=10.0$

Figure 6: Variation of velocity profile.

investigated. The following are concluded from the obtained results:

(i) The Ri and Ha significantly affect the heat transfer mechanism. Heat transfer rate increases with the increase of Ri. However, the convective flows become weaker and therefore the heat transfer rate decreases as $\mathrm{Ha}$ increases.

(ii) The recirculation eddy in the cavity is reduced with the existence of magnetic field, as the magnetic field strength increases with the decrease of the convection 


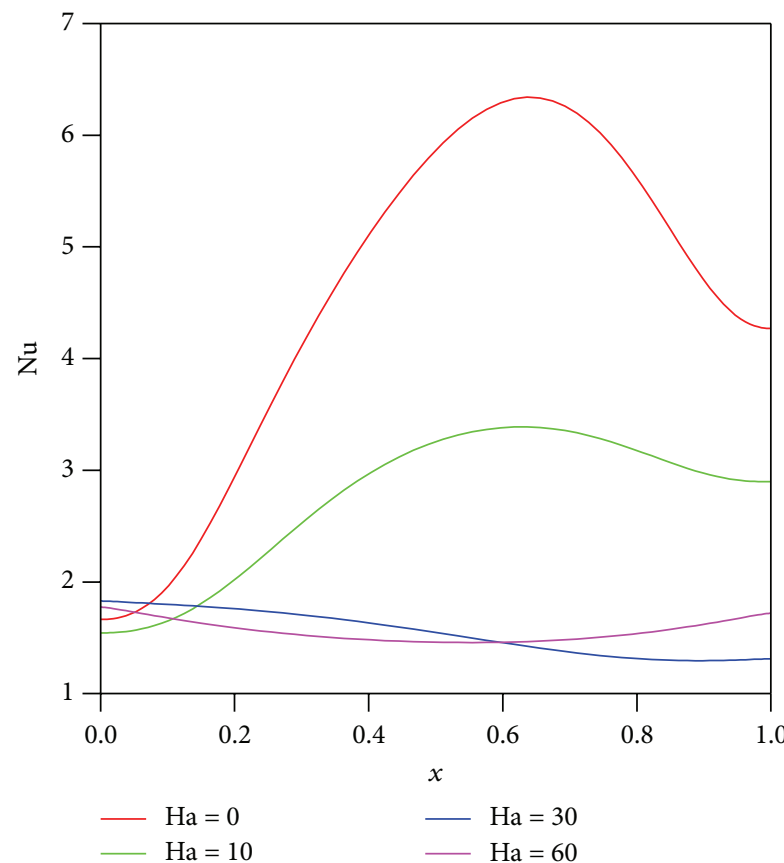

(a) $\mathrm{Ri}=0.1$

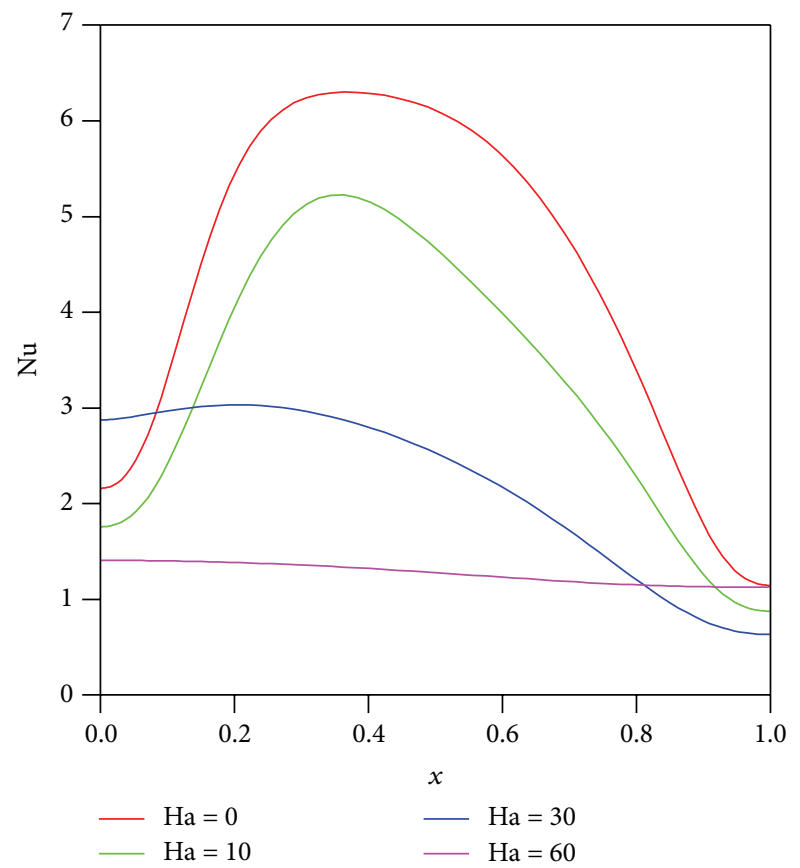

(b) $\mathrm{Ri}=1.0$

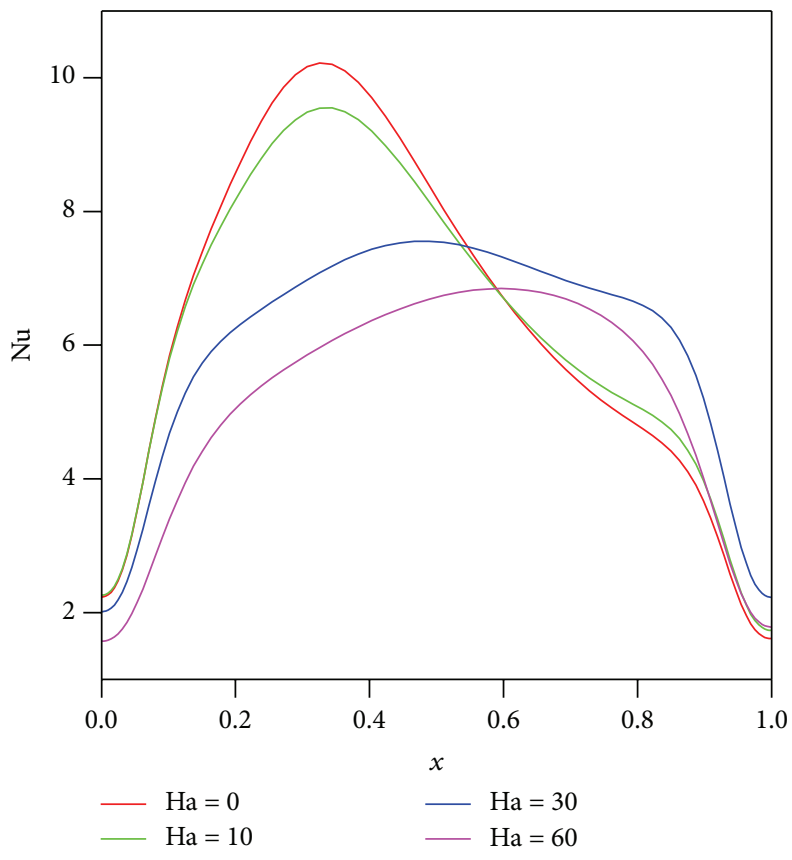

(c) $\mathrm{Ri}=10.0$

FIGURE 7: Variation of local Nusselt number.

heat transfer. Subsequently, conduction heat transfer is dominant.

\section{Nomenclature}

c: Specific heat capacity, $\mathrm{J} / \mathrm{kg} \mathrm{K}$

$g$ : Gravitational acceleration, $\mathrm{m} / \mathrm{s}^{2}$
Gr: Grashof number

$H$ : Height of the cavity, m

Ha: Hartmann number

$k$ : Thermal conductivity of fluid, W/m K

$\overline{\mathrm{Nu}}$ : Average Nusselt number 


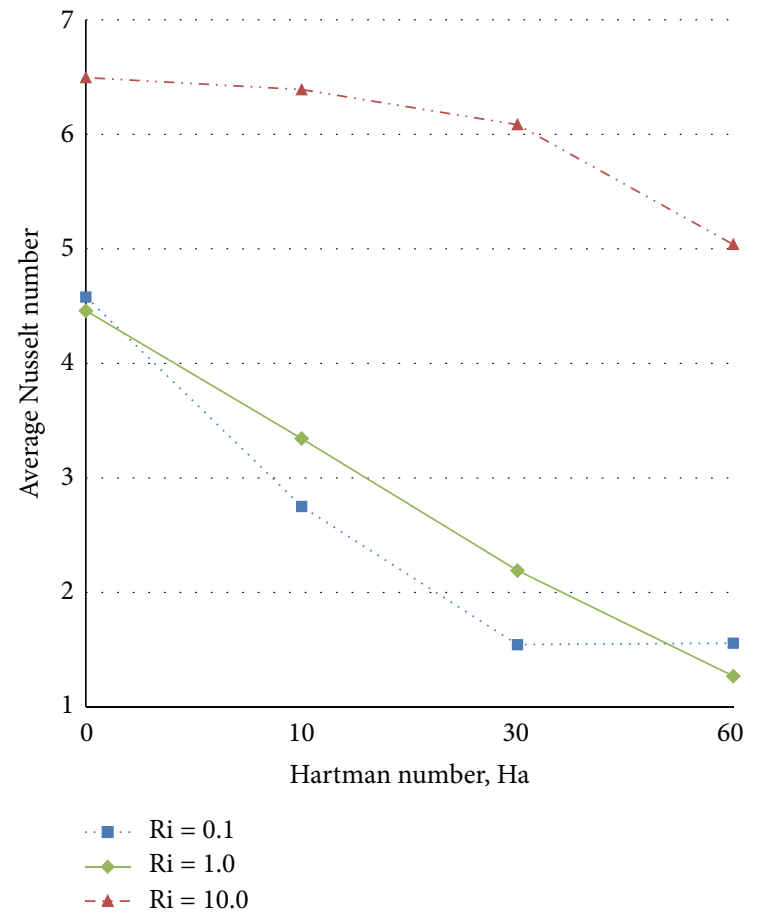

FIgURE 8: Effects of Hartmann number on average Nusselt number along the bottom wall for various Richardson number.

$\mathrm{Nu}_{x}$ : Local Nusselt number

$\mathrm{p}$ : $\quad$ Pressure, $\mathrm{Pa}$

$P: \quad$ Dimensionless pressure

Pr: Prandtl number

Re: Reynolds number

Ri: Richardson number

$t$ : Time

$T_{c}$ : $\quad$ Temperature at cold wall, ${ }^{\circ} \mathrm{C}$

$T_{h}$ : Temperature at hot wall, ${ }^{\circ} \mathrm{C}$

$u, v$ : Velocities in the $x$ - and $y$-directions, respectively

$U, V$ : Dimensionless velocity in $X$ - and $Y$-directions, respectively

$U_{0}$ : Lid velocity, $\mathrm{m} / \mathrm{s}$

$x, y$ : Cartesian coordinates

$X, Y$ : Dimensionless Cartesian coordinates.

\section{Greek Symbols}

$\alpha$ : Thermal diffusivity, $\mathrm{m}^{2} / \mathrm{s}$

$\beta$ : Coefficient of thermal expansion of fluid, $\mathrm{K}^{-1}$

$\theta$ : Dimensionless temperature

$\nu$ : Kinematic viscosity, $\mathrm{m}^{2} / \mathrm{s}$

$\mu$ : Dynamic viscosity, $\mathrm{kg} / \mathrm{ms}$

$\rho$ : Fluid density, $\mathrm{kg} / \mathrm{m}^{3}$

$\gamma$ : Inclination angle, degree

$\tau$ : Dimensionless time.

\section{Competing Interests}

The authors declare that they have no competing interests.

\section{Acknowledgments}

The authors would like to acknowledge the financial support received from the Universiti Tun Hussein Onn Malaysia (Grant nos. FRGS/1434 and GIPS/1201).

\section{References}

[1] K. Torrance, R. Davis, K. Eike et al., "Cavity flows driven by buoyancy and shear," Journal of Fluid Mechanics, vol. 51, no. 2, pp. 221-231, 1972.

[2] M. K. Moallemi and K. S. Jang, "Prandtl number effects on laminar mixed convection heat transfer in a lid-driven cavity," International Journal of Heat and Mass Transfer, vol. 35, no. 8, pp. 1881-1892, 1992.

[3] R. Iwatsu, J. M. Hyun, and K. Kuwahara, "Mixed convection in a driven cavity with a stable vertical temperature gradient," International Journal of Heat and Mass Transfer, vol. 36, no. 6, pp. 1601-1608, 1993.

[4] A. K. Prasad and J. R. Koseff, "Combined forced and natural convection heat transfer in a deep lid-driven cavity flow," International Journal of Heat and Fluid Flow, vol. 17, no. 5, pp. 460-467, 1996.

[5] K. M. Khanafer and A. J. Chamkha, "Mixed convection flow in a lid-driven enclosure filled with a fluid-saturated porous medium," International Journal of Heat and Mass Transfer, vol. 42, no. 13, pp. 2465-2481, 1999.

[6] H. F. Oztop and I. Dagtekin, "Mixed convection in two-sided lid-driven differentially heated square cavity," International Journal of Heat and Mass Transfer, vol. 47, no. 8-9, pp. 1761-1769, 2004.

[7] T. Basak, S. Roy, P. K. Sharma, and I. Pop, "Analysis of mixed convection flows within a square cavity with uniform and non-uniform heating of bottom wall," International Journal of Thermal Sciences, vol. 48, no. 5, pp. 891-912, 2009.

[8] T. Basak, S. Roy, P. K. Sharma, and I. Pop, "Analysis of mixed convection flows within a square cavity with linearly heated side wall(s)," International Journal of Heat and Mass Transfer, vol. 52, no. 9-10, pp. 2224-2242, 2009.

[9] T. S. Cheng and W.-H. Liu, "Effect of temperature gradient orientation on the characteristics of mixed convection flow in a lid-driven square cavity," Computers \& Fluids, vol. 39, no. 6, pp. 965-978, 2010.

[10] V. Sivakumar, S. Sivasankaran, P. Prakash, and J. Lee, "Effect of heating location and size on mixed convection in lid-driven cavities," Computers \& Mathematics with Applications, vol. 59, no. 9, pp. 3053-3065, 2010.

[11] D. S. Ducasse and P. Sibanda, "On mixed convection in a cavity with sinusoidally heated moving lid and uniformly heated and cooled side walls," Boundary Value Problems, vol. 2013, article 83, 2013.

[12] T. S. Cheng and W. H. Liu, "Effects of cavity inclination on mixed convection heat transfer in lid-driven cavity flows," Computers and Fluids, vol. 100, pp. 108-122, 2014.

[13] N. Rudraiah, R. M. Barron, M. Venkatachalappa, and C. K. Subbaraya, "Effect of a magnetic field on free convection in a rectangular enclosure," International Journal of Engineering Science, vol. 33, no. 8, pp. 1075-1084, 1995. 
[14] N. M. Al-Najem, K. M. Khanafer, and M. M. El-Refaee, "Numerical study of laminar natural convection in tilted enclosure with transverse magnetic field," International Journal of Numerical Methods for Heat and Fluid Flow, vol. 8, no. 6, pp. 651-672, 1998.

[15] H. F. Oztop, M. Oztop, and Y. Varol, "Numerical simulation of magnetohydrodynamic buoyancy-induced flow in a nonisothermally heated square enclosure," Communications in Nonlinear Science and Numerical Simulation, vol. 14, no. 3, pp. 770778, 2009.

[16] M. Pirmohammadi, M. Ghassemi, and G. A. Sheikhzadeh, "Effect of a magnetic field on buoyancy-driven convection in differentially heated square cavity," IEEE Transactions on Magnetics, vol. 45, no. 1, pp. 407-411, 2009.

[17] H. Nemati, M. Farhadi, K. Sedighi, H. R. Ashorynejad, and E. Fattahi, "Magnetic field effects on natural convection flow of nanofluid in a rectangular cavity using the Lattice Boltzmann model," Scientia Iranica B, vol. 19, no. 2, pp. 303-310, 2012.

[18] G. H. R. Kefayati, "Lattice Boltzmann simulation of MHD natural convection in a nanofluid-filled cavity with sinusoidal temperature distribution," Powder Technology, vol. 243, pp. 171-183, 2013.

[19] I. Mejri, A. Mahmoudi, M. A. Abbassi, and A. Omri, "MHD natural convection in a nanofluid-filled enclosure with nonuniform heating on both side walls," Fluid Dynamic \& Material Processing, vol. 10, no. 1, pp. 83-114, 2014.

[20] B. Ghasemi, S. M. Aminossadati, and A. Raisi, "Magnetic field effect on natural convection in a nanofluid-filled square enclosure," International Journal of Thermal Sciences, vol. 50, no. 9, pp. 1748-1756, 2011.

[21] M. Sheikholeslami and R. Ellahi, "Three dimensional mesoscopic simulation of magnetic field effect on natural convection of nanofluid," International Journal of Heat and Mass Transfer, vol. 89, Article ID 12118, pp. 799-808, 2015.

[22] A. J. Chamkha, "Hydromagnetic combined convection flow in a vertical lid-driven cavity with internal heat generation or absorption," Numerical Heat Transfer, Part A: Applications, vol. 41, no. 5, pp. 529-546, 2002.

[23] L. K. Saha, K. M. S. Uddin, and M. A. Taher, "Effect of internal heat generation or absorption on MHD mixed convection flow in a lid driven cavity," American Journal of Applied Mathematics, vol. 3, pp. 20-29, 2015.

[24] M. M. Rahman, M. A. Alim, and M. M. A. Sarker, "Numerical study on the conjugate effect of joule heating and magnatohydrodynamics mixed convection in an obstructed lid-driven square cavity," International Communications in Heat and Mass Transfer, vol. 37, no. 5, pp. 524-534, 2010.

[25] H. F. Oztop, K. Al-Salem, and I. Pop, "MHD mixed convection in a lid-driven cavity with corner heater," International Journal of Heat and Mass Transfer, vol. 54, no. 15-16, pp. 3494-3504, 2011.

[26] A. Malleswaran, S. Sivasankaran, and M. Bhuvaneswari, "Effect of heating location and size on MHD mixed convection in a liddriven cavity," International Journal of Numerical Methods for Heat \& Fluid Flow, vol. 23, no. 5, pp. 867-884, 2013.

[27] S. Sivasankaran, A. Malleswaran, J. Lee, and P. Sundar, "Hydromagnetic combined convection in a lid-driven cavity with sinusoidal boundary conditions on both sidewalls," International Journal of Heat and Mass Transfer, vol. 54, no. 1-3, pp. 512-525, 2011.
[28] R. Nasrin and S. Parvin, "Hydromagnetic effect on mixed convection in a lid-driven cavity with sinusoidal corrugated bottom surface," International Communications in Heat and Mass Transfer, vol. 38, no. 6, pp. 781-789, 2011.

[29] S. E. Ahmed, M. A. Mansour, and A. Mahdy, "MHD mixed convection in an inclined lid-driven cavity with opposing thermal buoyancy force: effect of non-uniform heating on both side walls," Nuclear Engineering and Design, vol. 265, pp. 938-948, 2013.

[30] G. H. R. Kefayati, M. Gorji-Bandpy, H. Sajjadi, and D. D. Ganji, "Lattice Boltzmann simulation of MHD mixed convection in a lid-driven square cavity with linearly heated wall," Scientia Iranica B, vol. 19, no. 4, pp. 1053-1065, 2012.

[31] K. Al-Salem, H. F. Öztop, I. Pop, and Y. Varol, "Effects of moving lid direction on MHD mixed convection in a linearly heated cavity," International Journal of Heat and Mass Transfer, vol. 55, no. 4, pp. 1103-1112, 2012.

[32] S. Udhayakumar, A. D. A. Rejeesh, T. V. S. Sekhar, and R. Sivakumar, "Numerical investigation of magnetohydrodynamic mixed convection over an isothermal circular cylinder in presence of an aligned magnetic field," International Journal of Heat and Mass Transfer, vol. 95, pp. 379-392, 2016.

[33] G. A. Sheikhzadeh, S. M. Sebdani, M. Mahmoodi, E. Safaeizadeh, and S. E. Hashemi, "Effect of a magnetic field on mixed convection of a nanofluid in a square cavity," Journal of Magnetics, vol. 18, no. 3, pp. 321-325, 2013.

[34] J. Rahmannezhad, A. Ramezani, and M. Kalteh, "Numerical investigation of magnetic field effects on mixed convection flow in a nanofluid-filled lid-driven cavity," International Journal of Engineering, Transactions A: Basics, vol. 26, no. 10, pp. 12131224, 2013.

[35] M. Muthtamilselvan and D.-H. Doh, "Magnetic field effect on mixed convection in a lid-driven square cavity filled with nanofluids," Journal of Mechanical Science and Technology, vol. 28, no. 1, pp. 137-143, 2014.

[36] M. M. Rashidi, M. Nasiri, M. Khezerloo, and N. Laraqi, "Numerical investigation of magnetic field effect on mixed convection heat transfer of nanofluid in a channel with sinusoidal walls," Journal of Magnetism and Magnetic Materials, vol. 401, pp. 159-168, 2016.

[37] A. Aghaei, H. Khorasanizadeh, G. Sheikhzadeh, and M. Abbaszadeh, "Numerical study of magnetic field on mixed convection and entropy generation of nanofluid in a trapezoidal enclosure," Journal of Magnetism and Magnetic Materials, vol. 403, pp. 133145, 2016.

[38] S. V. Patankar, Numerical Heat Transfer and Fluid Flow, Hemisphere Series on Computational Methods in Mechanics and Thermal Science, CRC Press, Washington, DC, USA, 1980. 

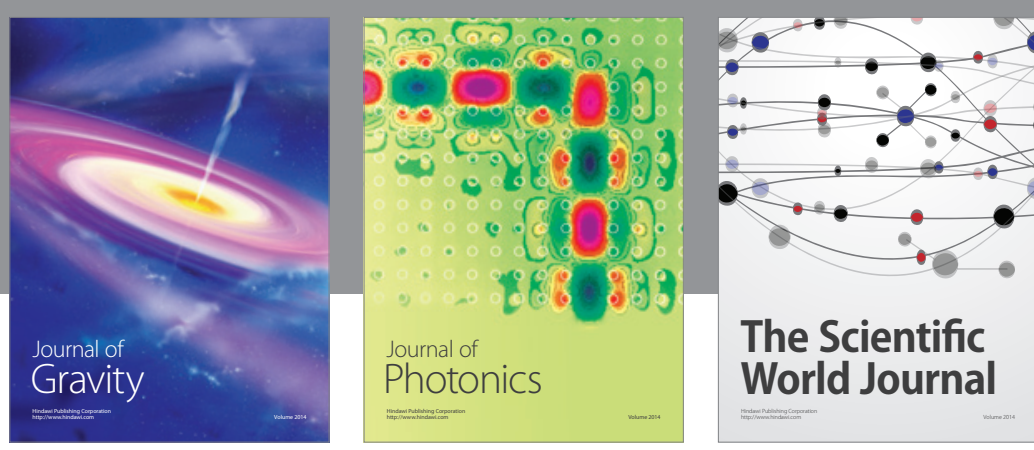

The Scientific World Journal
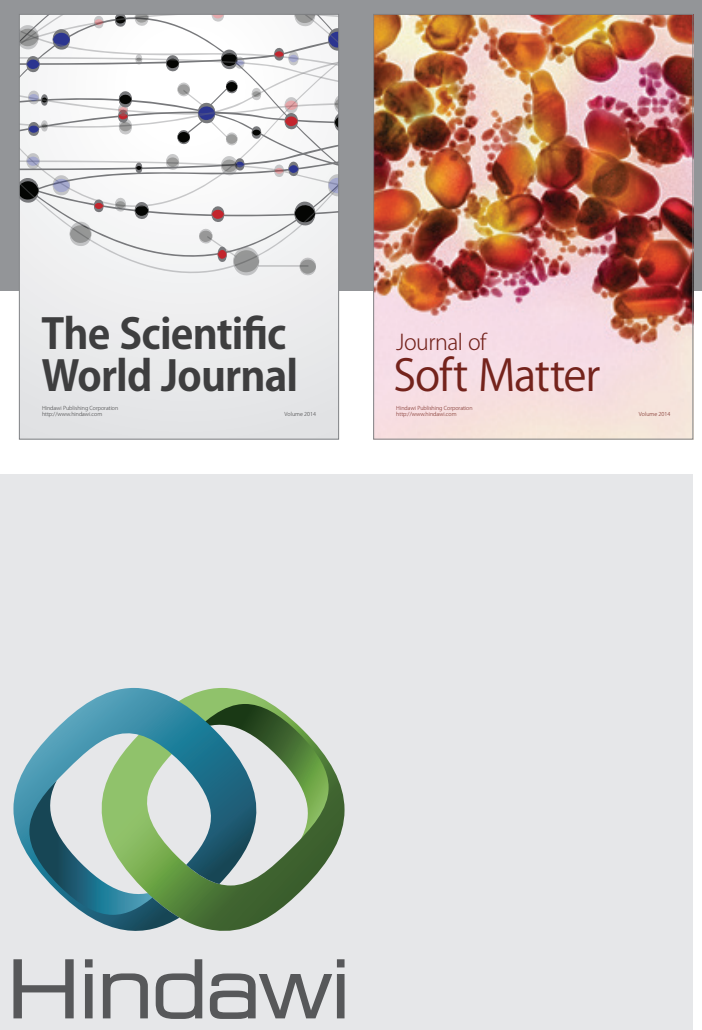

Submit your manuscripts at

http://www.hindawi.com

nternational Journal of

Statistical Mechanics
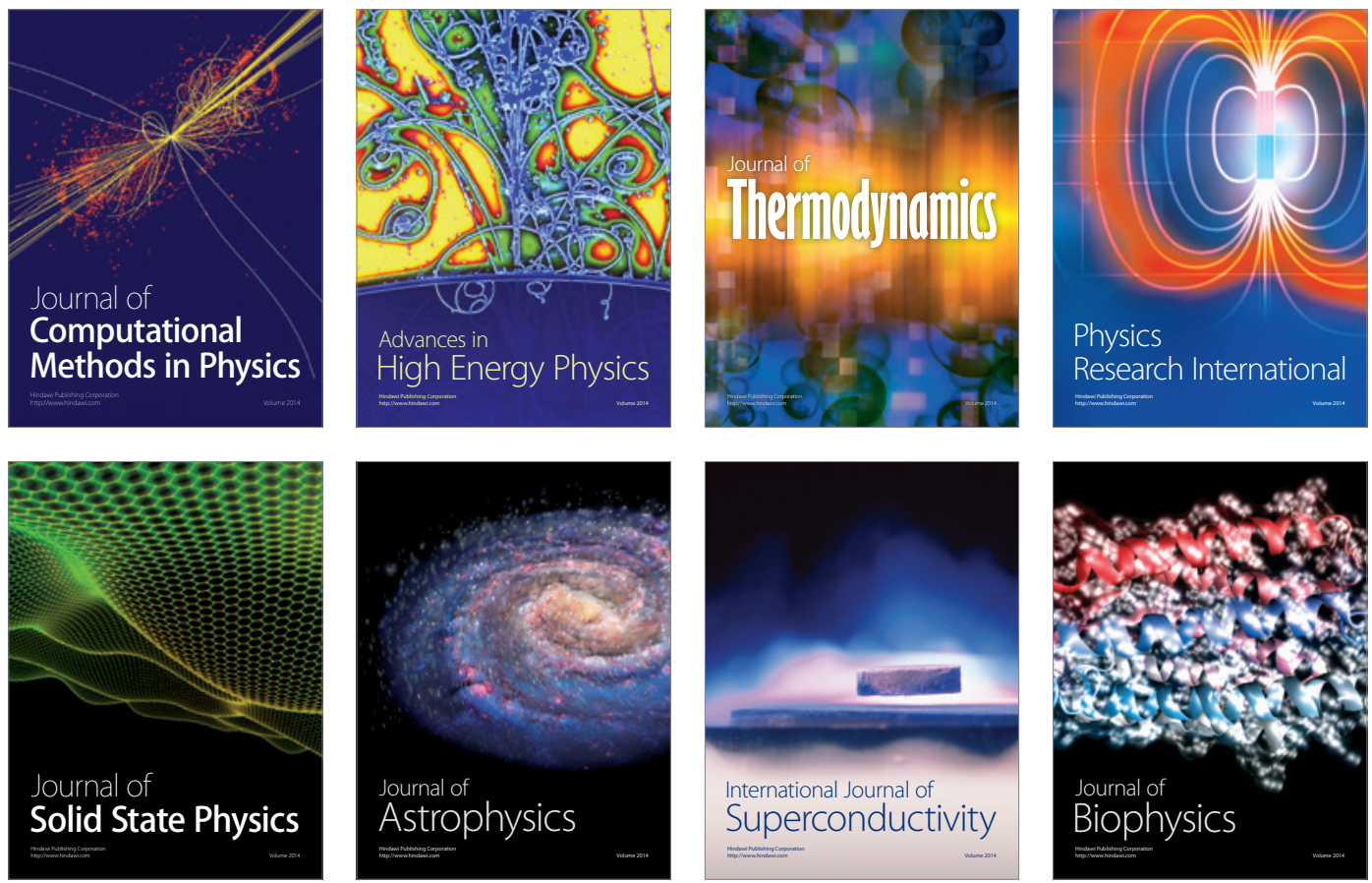
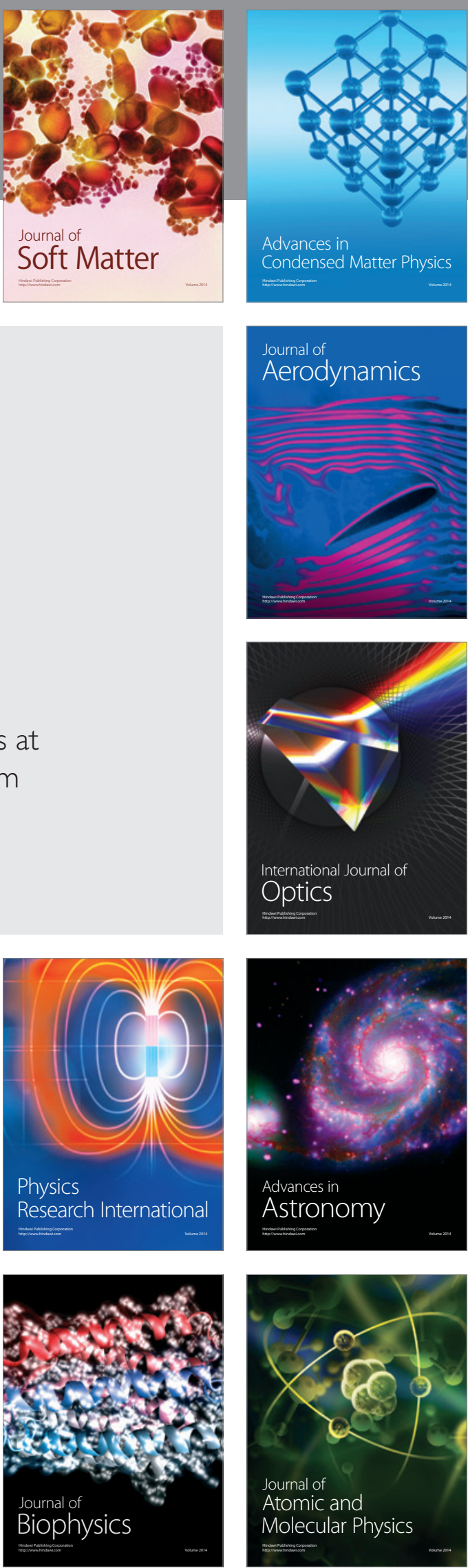